\title{
Alain Montandon, Théophile Gautier le poète impeccable
}

\section{Lise Sabourin}

\section{OpenEdition}

1 Journals

\section{Édition électronique}

URL : http://journals.openedition.org/studifrancesi/4425

DOI : 10.4000/studifrancesi.4425

ISSN : 2421-5856

\section{Éditeur}

Rosenberg \& Sellier

\section{Édition imprimée}

Date de publication : 1 septembre 2016

Pagination : 351-352

ISSN : 0039-2944

\section{Référence électronique}

Lise Sabourin, «Alain Montandon, Théophile Gautier le poète impeccable », Studi Francesi [En ligne], 179 (LX | II) | 2016, mis en ligne le 01 septembre 2016, consulté le 18 septembre 2020. URL : http:// journals.openedition.org/studifrancesi/4425; DOI : https://doi.org/10.4000/studifrancesi.4425

Ce document a été généré automatiquement le 18 septembre 2020.

\section{(c) (i) (9)}

Studi Francesi è distribuita con Licenza Creative Commons Attribuzione - Non commerciale - Non opere derivate 4.0 Internazionale. 


\title{
Alain Montandon, Théophile Gautier le poète impeccable
}

\author{
Lise Sabourin
}

\section{RÉFÉRENCE}

ALAIN MONTANDON, Théophile Gautier le poète impeccable, biographie, Paris, Éditions Aden, 2013, 523 pp.

1 Sans cesse en quête de beauté, étranger à l'esprit matérialiste, le poète magicien du verbe, médiateur de haute esthétique en une époque de démocratisation culturelle, Théophile Gautier a mené une existence essentiellement remplie par la fréquentation des littérateurs et des peintres, par la rédaction de ses chroniques dans les journaux, de ses romans à fondements souvent historiques, de sa poésie «impeccable» comme le dit le titre de cette biographie, essentiellement intellectuelle et esthétique. Alain Montandon s'attaque en effet à une tâche difficile: après ses prédécesseurs plus factuels (Senneville, Ubersfeld et Senninger) et la parution de la Correspondance (par Claudine Lacoste Veyssère chez Droz, 1985-2000, 12 volumes), il choisit de considérer essentiellement Gautier sous l'angle de la poétique de l'imaginaire. Ce n'est pas tout de parler, comme on l'y a souvent restreint, du «style coulant» de Gautier, encore faut-il en cerner les substrats esthétiques, les rencontres nourricières, les modalités créatives. Après un prologue (pp. 9-20), les sept chapitres suivent grosso modo les étapes de la vie, mais en scrutant en fait plutôt thématiquement l'évolution de Gautier.

2 «Premiers poèmes et amitiés» (pp. 21-84) le situe comme apprenti peintre contraint par sa myopie et la ruine de son père en 1848 à assurer la vie matérielle de sa famille par le journalisme, tout en s'insérant dans le milieu romantique au sein de la génération des Jeunes-France. Son compagnonnage avec Balzac (qu'il voit plus en visionnaire qu'en réaliste), son admiration pour Hugo (jamais démentie, malgré leurs divergences sur l'engagement artistique), sa proximité avec Nerval (dans l'angoisse du Doppelgänger) nourriront toute son œuvre. Ses rêves de jeunesse en manifestent les thèmes 
récurrents: le plaisir amical de converser sur l'Allemagne ou l'Orient, le goût de la retraite pour savourer l'apport des lectures et des rencontres, l'évasion par le voyage, l'envol de l'esprit au sein de la nature comme devant l'architecture gothique, le plaisir de retrouver le souvenir des fêtes galantes de Fragonard et Watteau, une conception de l'amour vampirique correspondant aux fantasmes d'une victime consentante.

Dans «Tristesse» (pp. 85-132), Alain Montandon sonde la mélancolie permanente de Gautier, deuil d'une espérance abolie, plutôt que simple nostalgie d'un bonheur dont il se sent exilé dès l'enfance. Tromperie des apparences, jeu des masques, souvenir d'un passé disparu provoquent un sentiment de flottement de l'âme, comme un vaisseau sur une mer indécise, mais sans espoir de résurrection si ce n'est par le rêve qu'est la création esthétique. La métamorphose, le passage, l'avatar sont les réponses à l'excessif besoin d'idéal qui provoque prostration, excitation et désir de mort. Pris entre le dégoût du monde et le besoin de sociabilité, le poète cherche la sublimation par le savoir et l'idéalisation par l'art.

4 «La Danse» (pp.133-175) rappelle les liens étroits que Gautier entretient avec les ballerines (l'éthérée Taglioni de La Sylphide, Elssler l'érotique païenne, l'aérienne Carlotta Grisi qui alliait force et grâce), mais aussi son regard, très intime et peu technique à la fois, sur le ballet. Lui qui composa tant de livrets pour cet art, dont huit montés de son vivant, il sait reconnaître dans la danse (qu'il s'agisse du ballet féerie qu'il préfère à tout ou des danses espagnoles comme la cachucha) le langage plastique de l'inconscient, ce «somnambulisme artificiel»(p.157), qui permet d'échapper au temps, à l'espace, à la pesanteur, dans un vertige éphémère qui conjure tout en la rappelant la suprême beauté de la mort.

«Feuilletons et salons» (pp. 177-245) fait le point sur l'activité journalistique de Gautier, qui, on le sait, tint de 1837 à sa mort en 1872, la chronique théâtrale de «La Presse», puis du «Moniteur universel», devenu «Journal officiel», enfin de «La Gazette de Paris». Ses mille quatre cents feuilletons le montrent souvent supportant mal cette «meule du journal», qui est sa source principale de revenus, soumis au rythme hebdomadaire pesant, sollicité constamment, mais préservant avec totale intégrité sa liberté de jugement, quitte à pratiquer la mansuétude de l'éloge avec feinte naïveté. Lui qui rêve d'un théâtre poétique à la Shakespeare, il doit rendre compte de vaudevilles dominants, de spectacles de cirque, et heureusement de concerts et d'opéras. «Être soi à travers les autres, difficulté immense!», reconnaît-il (cité p. 182), mais il y a trouvé sa plus grande notoriété, grâce à son analyse fine et ferme, à son aptitude à relever le niveau souvent médiocre par des stratégies d'écriture de contournement ou d'ironie piquante. Le découragement qu'il éprouve à se voir plus reconnu pour sa critique que pour ses poésies, pourtant réunies en édition complète avec España en 1845 et enrichie en 1852 d'Émaux et Camées, est compensé par son plaisir à rédiger ses Salons en 1834, 1836, 1837, 1847 et à commenter les Expositions Universelles de 1855 et 1867. Clairvoyant, pénétrant, érudit et ouvert, il sait offrir une description précise et élégante des tableaux qu'il voit, entrer dans le microcosme intérieur des artistes (notamment Delacroix, Doré, Corot), transposant parfois ses articles en poèmes et méditant sur le Beau. Restant platonicien sur le principe («la Beauté est la splendeur du Vrai»), il souligne surtout l'illusion du désir qui nous y pousse, sachant maintenir ses «contradictions irrésolues» (selon la formule de Stéphane Guégan citée p. 215) par la poursuite de ses fantasmes devant les toiles. L'art est bien pour lui un supplément d'existence répondant à la fêlure narcissique, une rêverie de puissance et de jouissance 
entre réalité et irréel, une fantaisie de l'imagination permettant d'atteindre cet «étrangement familier» qu'offrent les grands chefs-d'œuvre, de Léonard de Vinci par exemple, et qui seuls atteignent leur but de troubler et rendre rêveur.

«L'insatiable voyageur» (pp. 247-389) éprouve dès 1836, par sa découverte de la Belgique et de la Hollande avec Nerval, le besoin d'évasion, pour échapper au feuilleton, mais aussi au poids d'une existence trop déterminée pour sa marginalité naturelle. Pourtant Gautier n'est pas dupe de l'illusion du voyage, sachant y porter sa culture, mais refusant de transcrire les guides ou les relations de ses prédécesseurs. Il s'attache à narrer que ce qu'il voit, en une écriture réaliste mais subjective. Son goût des vues panoramiques, sa méfiance devant un progrès technique qui n'est pas forcément une avancée le rendent sensible aux détails, des mœurs, du vêtement comme de l'architecture, qui révèlent les peuples, dont il redoute déjà l'uniformisation par la vitesse des transports. Ébloui en Espagne par l'ascétisme morbide, la corrida et la «conscience du néant» de Goya, dès 1840 il contracte «la maladie du bleu» qui le conduira en Algérie en 1845, en Italie en 1850, puis à Constantinople en 1852, en cet Orient ensoleillé qui communie avec le désert. Mais son intérêt ethnologique et son goût des coloris lui feront aussi apprécier la Russie en 1858-59 et 1861.

7 Les «Femmes» (pp.391-437) sont nombreuses dans l'existence de Gautier, mais l'admiration esthétique pour la femme idéale n'empêche pas le mépris condescendant pour la maîtresse du moment. La Cydalise, Victorine, Eugénie Fort la délaissée (dont il reconnaît difficilement le fils, Toto), Alice Ozy qui le fascine physiquement, Marie Mattéi avec qui il file pourtant le doux amour à Venise ne supplanteront jamais les Grisi: après Giulia la cantatrice, sa cousine Carlotta, danseuse et chanteuse, garde une place centrale, même par rapport à sa sœur Ernesta, mère de ses deux filles Judith et Estelle, compagne finalement repoussée en 1866.

8 Les «Dernières années» (pp. 439-477) sont encore animées dans la maisonnée, entre ses sœurs, ses filles, ses amis, ses animaux familiers, mais Gautier, célèbre et enrichi sous le Second Empire, se sent vite vieillir et meurt atteint moralement comme physiquement par les privations de 1870-71. La conclusion (pp. 479-494), avant une chronologie et des indications bibliographiques, revient sur le statut de poète de Gautier: quoique mal apprécié pour sa poésie, jugée trop formelle et impassible, il possédait bien ce tempérament poétique, exprimé d'ailleurs aussi bien dans les autres genres littéraires qu'il pratiqua, qui cherche à protester de son écart avec la norme par la combinaison du visuel et du verbal, du plastique et du musical. 